\title{
Negative emotions affect postoperative scores for evaluating functional knee recovery and quality of life after total knee replacement
}

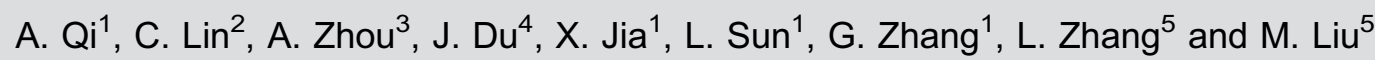 \\ ${ }^{1}$ Department of Nursing, Laiwu People's Hospital, Laiwu, China \\ ${ }^{2}$ Department of Cardiology, Laiwu People's Hospital, Laiwu, China \\ ${ }^{3}$ The First Ward of the Surgical Department, Laiwu People's Hospital, Laiwu, China \\ ${ }^{4}$ Department of Internal Medicine, Branch of Laiwu People's Hospital, Laiwu People's Hospital, Laiwu, China \\ ${ }^{5}$ Department of Traumatic Orthopedics, Shengjing Hospital, China Medical University, Shenyang, China
}

\begin{abstract}
This study aimed to determine whether psychological factors affect health-related quality of life (HRQL) and recovery of knee function in total knee replacement (TKR) patients. A total of 119 TKR patients (male: 38; female: 81) completed the Beck Anxiety Inventory (BAI), Beck Depression Inventory (BDI), State Trait Anxiety Inventory (STAI), Eysenck Personality Questionnairerevised (EPQR-S), Knee Society Score (KSS), and HRQL (SF-36). At 1 and 6 months after surgery, anxiety, depression, and KSS scores in TKR patients were significantly better compared with those preoperatively $(P<0.05)$. SF-36 scores at the sixth month after surgery were significantly improved compared with preoperative scores $(P<0.001)$. Preoperative Physical Component Summary Scale (PCS) and Mental Component Summary Scale (MCS) scores were negatively associated with extraversion (E score) $(B=-0.986$ and -0.967 , respectively, both $\mathrm{P}<0.05)$. Postoperative $P C S$ and State Anxiety Inventory (SAI) scores were negatively associated with neuroticism ( $N$ score; $B=-0.137$ and -0.991 , respectively, both $P<0.05$ ). Postoperative MCS, SAI, Trait Anxiety Inventory (TAI), and BAI scores were also negatively associated with the $\mathrm{N}$ score $(\mathrm{B}=-0.367,-0.107$, -0.281 , and -0.851 , respectively, all $\mathrm{P}<0.05)$. The KSS function score at the sixth month after surgery was negatively associated with TAl and $\mathrm{N}$ scores $(B=-0.315$ and -0.532 , respectively, both $P<0.05$ ), but positively associated with the $E$ score $(B=0.215, P<0.05)$. The postoperative KSS joint score was positively associated with postoperative $P C S(B=0.356, P<0.05)$. In conclusion, for TKR patients, the scores used for evaluating recovery of knee function and HRQL after 6 months are inversely associated with the presence of negative emotions.
\end{abstract}

Key words: Total knee replacement; Negative emotion; Anxiety; Depression; Functional recovery; Health-related quality of life

\section{Introduction}

Osteoarthritis $(\mathrm{OA})$ is a debilitating degenerative joint disease that is characterized by underlying erosion of articular cartilage and subchondral bone (1). The knee joints are among the earliest and most frequent joints involved in OA. OA patients suffer from considerable functional impairment of the knee joint and severe chronic pain. Among the available treatments, total knee replacement (TKR) is the safest and most cost-effective method to alleviate pain and to restore function of the knee joint in OA patients $(2,3)$. Despite these advantages, many TKR patients still have residual knee impairment and functional limitations as compared with age-matched controls. One year after TKR, patients walk $15 \%$ slower than age-matched healthy individuals with no known knee pathologies $(4,5)$. Interestingly, previous studies have shown that complications associated with TKR are not due to failed surgical procedures but might be closely related to the patient's emotional health or to other long-term or perioperative psychological factors $(6,7)$.

Although TKR is a safe and highly successful procedure in OA patients, some patients suffer from persistent pain and exhibit only partial recovery after TKR (8). Many patients who choose surgery experience negative moods preoperatively, including anxiety, depression and fear, in anticipation of potential surgery-related complications or death, as well as anxiety about the postoperative recovery phase (9). A previous study reported that patients with distressed mental states postoperatively had a greater risk of continued physical disability (10). Furthermore, mental distress resulting from negative emotions is associated with

Correspondence: Guiqin Zhang: <zhangguiqin0403@163.com> | Min Liu<liumin1023@yeah.net> 
greater difficulty during recovery after TKR, with sharper pain levels and greater limitations of joint function and mobility (11). Therefore, in a subset of patients, negative emotions increase the risk of poor outcomes, including decreased health-related quality of life (HRQL) and increased severity of physical disability and discomfort (12). Recurrent pain after surgery is a frequent, and sometimes severe, health problem that significantly affects the HRQL of OA patients (13). Accordingly, besides better preparing patients for the experience of surgery, potentially, a major improvement in outcomes could be achieved if patients could avoid preoperative and postoperative distress and depression (9). Therefore, the present study evaluated the effect of negative emotions, such as anxiety, depression, and personality factors, on HRQL and recovery of knee joint function in patients after TKR. We anticipate that this study will contribute to identify more effective ways to overcome the challenges encountered by patients during postoperative recovery and to accelerate their social re-adaptation (especially on a psychological level), thus improving their HRQL.

\section{Material and Methods}

\section{Ethics statement}

This study was performed after obtaining approval from the Institutional Review Board of Laiwu People's Hospital. Informed written consent was obtained from each eligible participant, and the study was conducted according to the Declaration of Helsinki (14).

\section{Study subjects}

A questionnaire-based survey was conducted on patients who underwent unilateral TKR in the Department of Surgery of Laiwu People's Hospital between February 2013 and January 2014. The inclusion criteria for patients in this study were as follows: 1) patients who underwent TKR for $O A$, degenerative $O A$, or rheumatoid arthritis; 2) no history of knee surgery or hip surgery; 3) no psychiatric history; 4) written informed consent was available; and 5) all questionnaires were completed. A total of 135 eligible patients were enrolled in this study and were asked to complete extensive questionnaires upon admission within 1 week before surgery. After surgery, the same questionnaires were mailed to each patient at 1 and 6 months after surgery to assess short-term and long-term postoperative treatment effects, respectively. A total of 119 patients (38 men and 81 women; mean age, 62.1 \pm 10.12 years old) completed all of the required questionnaires to be eligible for inclusion in this study. The entire study was designed as a prospective trial with consecutive patients, and a comparative study was also performed based on the preoperative and postoperative treatment effects in the patients.

\section{Questionnaires}

General information that was collected from the patients included age, gender, marital status, degree of education, average monthly income, occupation, and source of medical expenses. The following questionnaires were also conducted to collect study-related patient information. 1) The State-Trait Anxiety Inventory (STAI), which has a total of 40 items that are mainly used for evaluation of trait anxiety. The STAI includes a State Anxiety Inventory (SAI), which is used for evaluating short-term anxiety, and a Trait Anxiety Inventory (TAI), which is used for evaluating longterm anxiety. The maximum score on this questionnaire is 80 and the minimum score is 20 (15). 2) The Beck Anxiety Inventory (BAI), which is mainly used for the evaluation of anxiety states, includes 21 different anxiety symptoms. Participants must give the personal effects of each symptom score from 0 (no effect) to 3 (severe effects) (16). The Beck Depression Inventory (BDI) includes 21 self-assessments of depression symptoms, and each question has four selfassessment indices to reflect the degree of depression: score $<11$, no depression; score ranging from 11 to 15 , potential depressive symptoms; score $>16$, depression; and score $>25$, severe depression (17). 3) The Eysenck Personality Questionnaire-Revised (EPQR-S) includes four parts as follows: extraversion or introversion (E); neuroticism $(\mathrm{N})$; psychoticism $(\mathrm{P})$, and lying and dissimulation $(\mathrm{L})$. The standard T score $=50+10 \times$ (original score of the participant mean score of all participants)/standard deviation (18). 4) The Knee Society Score (KSS) includes a joint score (pain score of 50 , activity range score of 25 , and stability score of 25) and joint function score (climbing the stairs score of 50 and walking distance score of 50) $(19,20)$. 5) The Short-Form Health Survey questionnaire (SF-36) (Chinese version) was used to evaluate the patients' HRQL, which included body pain (BP), physical functioning (PF), physical role limitation (RP), emotional role limitation (RE), mental health $(\mathrm{MH})$, social functioning (SF), vitality (VT), general health $(\mathrm{GH})$, a Physical Component Summary Scale (PCS), and a Mental Component Summary Scale (MCS). The range in score for each part was $0-100$, directly reflecting the participants' health conditions (21). The patients' psychological state and activity function 1 week before surgery were evaluated by the BAI, BDI, STAI, EPQR-S, KSS, and SF-36. The patients completed the $\mathrm{BAI}, \mathrm{BDI}, \mathrm{KSS}$, and SF-36 questionnaires at 1 and 6 months postoperatively, and the scores of each scale were calculated.

\section{Statistical analysis}

Statistical analysis was performed with the SPSS software, version 18.0 (USA). Data are reported as means \pm SD. The differences in scores (preoperatively and postoperatively) were evaluated using the paired $t$-test. The associations of psychological and psychic factors with HRQL and KSS scores were analyzed by performing multiple linear regression using the enter method, which simultaneously adds all of the variables to the model. Statistical results were considered significant when the $P$ value was less than 0.05 . 


\section{Results}

\section{Scale scores before and after surgery}

The results of the STAI are reported in Table 1 . The preoperative scores of the SAI and TAI were $44.52 \pm 10.12$ and $43.61 \pm 9.23$, respectively. The average scores were 49.39 (P score), 49.45 (E score), 55.68 ( $\mathrm{N}$ score), and 48.11 ( $\mathrm{L}$ score). The preoperative score for the BAI was $38.15 \pm 8.42$. The BAl scores at the 1 st month after surgery and at the sixth month after surgery were $32.16 \pm 6.48$ and $28.69 \pm 7.52$, respectively. BAl scores 1 month after surgery were significantly lower than preoperative scores $(P<0.05)$. BAl scores 6 months after surgery were significantly lower than BAl scores 1 month after surgery $(P<0.05)$. The preoperative $\mathrm{BDI}$ score was $10.25 \pm 4.26$. BDI scores 1 and 6 months after surgery were $7.36 \pm 4.05$ and $5.45 \pm 3.12$, respectively. BDI scores 1 month after surgery were significantly lower than preoperative scores $(P<0.05)$. BDI scores 6 months after surgery were significantly lower than BDI scores 1 month after surgery $(P<0.05)$. KSS function scores 1 and 6 months after surgery were significantly improved compared with the preoperative scores (both $\mathrm{P}<0.05$ ).

\section{Evaluation of HRQL}

Table 2 shows the eight life aspects (BP, PF, RP, RE, $\mathrm{MH}, \mathrm{SF}, \mathrm{VT}$, and $\mathrm{GH}$ ) of the SF-36 score 6 months after surgery. The patients' SF-36 scores 6 months after surgery in these eight aspects were significantly higher than preoperative scores $(P<0.001)$. The PCS and MCS scores 6 months after surgery were higher than those preoperatively $(\mathrm{P}<0.001)$.
Effects of psychological and psychic factors on HRQL

Multiple linear regression analysis was performed with PCS and MCS as dependent variables and SAI, TAI, BAI, $B D I, P, E, N$, and $L$ as independent variables. Regression analysis showed that preoperative PCS and MCS scores were negatively associated with the $E$ score $(B=-0.986$, $\mathrm{B}=-0.967$, respectively, both $\mathrm{P}<0.05)$. Postoperative PCS and SAI scores were also negatively associated with the $E$ score $(B=-0.137, B=-0.991$, respectively, both $\mathrm{P}<0.05$ ). Postoperative MCS, SAI, TAI, and BAI scores were negatively associated with the $N$ score $(B=-0.367$, $B=-0.107, B=-0.281, B=-0.851$, respectively, all $P<0.05$; Table 3).

\section{Associations of psychological and psychic factors with the KSS score}

Multiple linear regression analysis was performed with knee joint and function scores 6 months after surgery as the dependent variables and SAI, TAI, BAI, BDI, P, E, $N, L, P C S$, and MCS as independent variables. Regression analysis showed that knee function 6 months after surgery was negatively associated with TAl and $\mathrm{N}$ scores $(B=-0.315, B=-0.532$, respectively, both $P<0.05)$, but positively associated with the $E$ score $(B=0.215, P<0.05)$. The KSS joint score was positively associated with the postoperative PCS score $(B=0.356, P<0.05$; Table 4).

\section{Discussion}

One of the main results of the present study was reduced strength of patients exhibiting negative emotions

Table 1. Scores of various psychological scales before and after surgery.

\begin{tabular}{lccc}
\hline & 1 week before surgery & 1 month after surgery & 6 months after surgery \\
\hline STAI & & & - \\
SAI & $44.52 \pm 10.12$ & - & - \\
TAI & $43.61 \pm 9.23$ & - & $28.69 \pm 7.52^{\star \#}$ \\
BAI & $38.15 \pm 8.42$ & $32.16 \pm 6.48^{*}$ & $5.45 \pm 3.12^{\star \#}$ \\
BDI & $10.25 \pm 4.26$ & $7.36 \pm 4.05^{*}$ & - \\
EPQR-S & & & - \\
E & $49.45 \pm 9.36$ & - & - \\
N & $55.68 \pm 10.02$ & - & - \\
P & $49.39 \pm 7.28$ & - & $88.26 \pm 14.63^{\star \#}$ \\
L & $48.11 \pm 6.19$ & & $82.70 \pm 20.38^{\star \#}$ \\
KSS score & & & \\
Knee joint score & $26.54 \pm 16.85$ & $65.47 \pm 19.12 .12^{*}$ & \\
Knee function score & $45.24 \pm 18.67$ &
\end{tabular}

STAI: State-Trait Anxiety Inventory; SAI: state anxiety inventory; TAI: trait anxiety inventory; BAI: Beck Depression Inventory; BDI: Beck Anxiety Inventory; EPQR-S: Eysenck Personality Questionnaire-revised; $\mathrm{E}$ : extraversion or introversion; N: neuroticism; P: psychoticism; L: lying and dissimulation; KSS: Knee Society Score. * $\mathrm{P}<0.05$, compared to before surgery; ${ }^{\#} \mathrm{P}<0.05$, compared to 1 month after surgery (paired $t$-test). 
Table 2. SF-36 scores before and after surgery.

\begin{tabular}{|c|c|c|c|c|c|c|c|}
\hline \multirow[t]{2}{*}{ Items } & \multicolumn{3}{|c|}{1 week before surgery } & \multicolumn{3}{|c|}{6 months after surgery } & \multirow[t]{2}{*}{$\mathrm{P}$} \\
\hline & Means $\pm S D$ & Median & $25-75 \%$ & Means \pm SD & Median & $25-75 \%$ & \\
\hline PF & $25.1 \pm 11.4$ & 23.1 & $16.0-37.0$ & $51.3 \pm 12.1$ & 52.1 & $40.0-60.0$ & $<0.001$ \\
\hline RP & $4.5 \pm 9.7$ & 0.0 & $0.0-0.0$ & $41.8 \pm 18.5$ & 44.0 & $28.0-65.0$ & $<0.001$ \\
\hline RE & $22.1 \pm 23.8$ & 17.8 & $0.0-35.3$ & $56.2 \pm 25.8$ & 59.2 & $32.5-87.5$ & $<0.001$ \\
\hline VT & $44.3 \pm 13.2$ & 40.2 & $34.0-56.0$ & $64.9 \pm 14.2$ & 66.0 & $51.0-81.0$ & $<0.001$ \\
\hline $\mathrm{MH}$ & $64.2 \pm 8.9$ & 63.9 & $55.0-73.0$ & $72.4 \pm 8.1$ & 73.2 & $63.5-83.0$ & $<0.001$ \\
\hline SF & $45.1 \pm 11.2$ & 49.5 & $41.0-60.0$ & $62.1 \pm 13.5$ & 65.2 & $44.0-86.0$ & $<0.001$ \\
\hline $\mathrm{BP}$ & $36.2 \pm 9.8$ & 38.1 & $37.5-49.8$ & $71.3 \pm 11.1$ & 74.0 & $60.5-87.5$ & $<0.001$ \\
\hline $\mathrm{GH}$ & $48.3 \pm 11.5$ & 45.6 & $39.0-59.5$ & $66.4 \pm 12.6$ & 68.0 & $48.2-88.0$ & $<0.001$ \\
\hline PCS & $28.2 \pm 5.8$ & 27.9 & $22.8-32.6$ & $57.2 \pm 9.9$ & 56.0 & $50.0-65.0$ & $<0.001$ \\
\hline MCS & $44.1 \pm 9.9$ & 43.9 & $34.2-52.8$ & $64.3 \pm 12.4$ & 65.0 & $51.3-78.7$ & $<0.001$ \\
\hline
\end{tabular}

PF: physical functioning; RP: physical role limitation; RE: emotional role limitation; VT: vitality; MH: mental health; SF: social functioning; BP: body pain; GH: general health; PCS: Component Summary Scale; MCS: Mental Component Summary Scale. The paired $t$-test was used for statistical analyses.

of depression and anxiety after TKR. Similar to patients with other diseases undergoing elective surgery, OA patients often experience high levels of anxiety before surgery because of the possibility of surgery-related complications or death. The use of surgical instruments and the thought of tissue penetration also often provoke fear and discomfort, and have been mentally associated with pain, destruction of body shape, and death (22). Furthermore, fear of neglect by society and family during the recovery phase, and patients' inferiority complexes and annoyance with discomfort can also lead to negative emotions such as depression, anxiety, paranoia, and fear (23). TKR is a safe surgical option for OA patients, but only a small proportion of the patient population show complete postoperative improvement in physical function, pain, and quality of life $(24,25)$. Previous studies have suggested that psychological factors contribute to poor outcomes after TKR $(26,27)$. Our study suggested that patients who underwent TKR showed excellent recovery of knee function and improvement in HRQL, but the scores used for evaluating recovery of knee function and HRQL after 6 months were inversely associated with the presence of negative emotions. We demonstrated that HRQL in patients at 6 months after unilateral TKR was significantly improved. However, based on our results, improvement in the MCS was less than the observed improvement in the PCS. Although improvement in physical function is the main factor contributing to patients' satisfaction with TKR, postoperative changes observed in the mental and emotional states are significantly different

Table 3. Multiple linear regression analysis of psychological and psychic factors with health-related quality of life.

\begin{tabular}{lccccc}
\hline & \multicolumn{2}{c}{ Before surgery } & & \multicolumn{2}{c}{ After surgery } \\
\cline { 2 - 3 } \cline { 6 - 6 } & PCS - Beta & MCS - Beta & & PCS - Beta & MCS - Beta \\
\hline SAI & -0.007 & -0.007 & & $-0.137^{*}$ & $-0.367^{*}$ \\
TAI & -0.005 & -0.005 & & 0.012 & $-0.107^{*}$ \\
BAI & -0.259 & -0.264 & & -0.017 & $-0.281^{*}$ \\
BDI & 0.243 & 0.247 & & 0.016 & 0.003 \\
P & 9.869 & -0.989 & & 11.689 & -0.361 \\
E & -14.233 & -14.365 & & 14.094 & -8.167 \\
N & $-0.986^{*}$ & $-0.967^{*}$ & & $-0.991^{*}$ & $-0.851^{*}$ \\
L & -0.002 & 0.003 & & -0.005 & 0.006 \\
\hline
\end{tabular}

PCS: Component Summary Scale; MCS: Mental Component Summary Scale. SAI: state anxiety inventory; TAl: trait anxiety inventory; BAl: Beck Depression Inventory; BDI: Beck Anxiety Inventory; P: psychoticism; E: extraversion or introversion; N: neuroticism; L: lying and dissimulation. ${ }^{*} \mathrm{P}<0.05$ (multiple linear regression analysis). 
Table 4. Multiple linear regression analysis of the Knee Society Score at 6 months after surgery and related scale scores.

\begin{tabular}{lcc}
\hline & Function score - Beta & Joint score - Beta \\
\hline SAI & 0.013 & -1.421 \\
TAI & $-0.315^{*}$ & 0.485 \\
BAI & -0.104 & -0.15 \\
BDI & -0.171 & 0.109 \\
P & -0.106 & -0.011 \\
E & $0.215^{*}$ & -0.211 \\
N & $-0.532^{*}$ & -0.418 \\
L & 0.151 & -0.492 \\
PCS & 0.129 & $0.356^{*}$ \\
MCS & -0.119 & 0.01 \\
\hline
\end{tabular}

SAl: state anxiety inventory; TAl: trait anxiety inventory; BAI: Beck Depression Inventory; BDI: Beck Anxiety Inventory; P: psychoticism; E: extraversion or introversion; $\mathrm{N}$ : neuroticism; L: lying and dissimulation; PCS: Component Summary Scale; MCS: Mental Component Summary Scale. ${ }^{*} \mathrm{P}<0.05$ (multiple linear regression analysis).

than the preoperative condition (28). A previous study showed that patients with preoperative anxiety or depression were less satisfied with TKR (29). Consequently, chronic pain or negative emotions in patients postoperatively might be a risk factor for decreased function rehabilitation, affecting the efficacy of TKR.

Negative emotions, including neurotic mood, anxiety, and depression, could affect improvement in HRQL. The present study showed that PCS and MCS scores were negatively associated with the neurotic value. This finding indicates that a lower level of HRQL might be associated with the personality traits of each patient. Patients with high neuroticism scores on the EPQ might have mental illnesses. High neuroticism is one of the risk factors for depression (30). Additionally, mental health affects TKR outcomes, and neurotic patients are at increased risk for physical disability after surgery because negative emotions have side effects that affect recovery of body function (10). Consequently, patients with anxiety and depression might require a longer rehabilitation time, which could affect their quality of life (31).

\section{References}

1. Goldring SR, Scanzello CR. Plasma proteins take their toll on the joint in osteoarthritis. Arthritis Res Ther 2012; 14: 111, doi: 10.1186/ar3741.

2. Leskinen J, Eskelinen A, Huhtala H, Paavolainen P, Remes V. The incidence of knee arthroplasty for primary osteoarthritis grows rapidly among baby boomers: a population-based study in Finland. Arthritis Rheum 2012; 64: 423-428, doi: 10.1002/ art.33367.

3. McClelland J, Zeni J, Haley RM, Snyder-Mackler L. Functional and biomechanical outcomes after using biofeedback for retraining symmetrical movement patterns after total knee
Moreover, negative thought patterns, hypervigilance, fear, and avoidance could result in physical deterioration, based on the fear-avoidance model of chronic pain (9). Therefore, postoperative recovery and disease severity are negatively affected in patients with high negative emotion levels after TKR. The findings of this study are consistent with previous studies showing that high levels of anxiety and depression predict poorer quality of life and increase the severity of pain after surgery $(11,32,33)$.

Our results strongly suggest that counseling of patients is urgently required to promote positive emotions and to improve outcomes. Satisfaction with in-hospital patient care could positively affect patients' moods, and caregivers' performance directly influences the quality of daily care (28). Therefore, daily care should be improved to enhance the positive emotions of patients. Additionally, postoperative pain is a major factor causing negative moods. Therefore, immediate and effective relief of postoperative pain might reduce anxiety and depression (34). Targeted and personalized rehabilitation, exercise, and sports programs might also help in improving knee function, mobility, and the moods of patients (8).

There are several limitations in this study. First, the number of patients in our study was small. Second, other variables, such as comorbidities or environmental factors, could have affected HRQL, but we failed to analyze these factors because of insufficient information. Finally, all of the enrolled patients were selected from a hospital setting in a specific geographical region. Therefore, the results might only be representative of this group of patients, and different results might be obtained in other geographical areas. However, this study provided important information about the factors affecting patients' outcomes and satisfaction after TKR.

In conclusion, recovery of knee function and HRQL in OA patients is significantly improved after TKR. However, the postoperative scores used for evaluating recovery of knee function and HRQL are affected by negative emotions, including neuroticism, anxiety, and depression. The finding that negative mood has an inverse correlation with recovery of knee function and quality of life warrants further examination. arthroplasty: a case report. J Orthop Sports Phys Ther 2012; 42: 135-144, doi: 10.2519/jospt.2012.3773.

4. Stevens-Lapsley JE, Schenkman ML, Dayton MR. Comparison of self-reported knee injury and osteoarthritis outcome score to performance measures in patients after total knee arthroplasty. PM R 2011; 3: 541-549.

5. Stevens-Lapsley JE, Bade MJ, Shulman BC, Kohrt WM, Dayton MR. Minimally invasive total knee arthroplasty improves early knee strength but not functional performance: a randomized controlled trial. J Arthroplasty 2012; 27: 1812-1819, doi: 10.1016/j.arth.2012.02.016. 
6. Sasaki E, Tsuda E, Yamamoto Y, Meada S, Otsuka H, Ishibashi Y. Relationship between patient-based outcome score and conventional objective outcome scales in postoperative total knee arthroplasty patients. Int Orthop 2014; 38: 373-378, doi: 10.1007/s00264-013-2064-5.

7. Odland AN, Callaghan JJ, Liu SS, Wells CW. Wear and lysis is the problem in modular TKA in the young OA patient at 10 years. Clin Orthop Relat Res 2011; 469: 41-47, doi: 10.1007/ s11999-010-1429-y.

8. Hirschmann MT, Testa E, Amsler F, Friederich NF. The unhappy total knee arthroplasty (TKA) patient: higher WOMAC and lower KSS in depressed patients prior and after TKA. Knee Surg Sports Traumatol Arthrosc 2013; 21: 2405-2411, doi: 10.1007/s00167-013-2409-z.

9. Cremeans-Smith JK, Greene K, Delahanty DL. Symptoms of postsurgical distress following total knee replacement and their relationship to recovery outcomes. J Psychosom Res 2011; 71: 55-57, doi: 10.1016/j.jpsychores.2010.12.002.

10. Clement ND, MacDonald D, Burnett R. Primary total knee replacement in patients with mental disability improves their mental health and knee function: a prospective study. Bone Joint J 2013; 95-B: 360-366.

11. Blackburn J, Qureshi A, Amirfeyz R, Bannister G. Does preoperative anxiety and depression predict satisfaction after total knee replacement? Knee 2012; 19: 522-524, doi: 10.1016/j.knee.2011.07.008.

12. O'Donnell ML, Creamer M, Elliott P, Atkin C, Kossmann T. Determinants of quality of life and role-related disability after injury: impact of acute psychological responses. J Trauma 2005; 59: 1328-1334.

13. van Hanswijck de Jonge $P$, Lloyd A, Horsfall $L$, Tan R, O'Dwyer PJ. The measurement of chronic pain and healthrelated quality of life following inguinal hernia repair: a review of the literature. Hernia 2008; 12: 561-569, doi: 10.1007/s10029008-0412-y.

14. PN M. World Medical Association publishes the Revised Declaration of Helsinki. Natl Med J India 2014; 27: 56.

15. Gros DF, Antony MM, Simms LJ, McCabe RE. Psychometric properties of the State-Trait Inventory for Cognitive and Somatic Anxiety (STICSA): comparison to the State-Trait Anxiety Inventory (STAI). Psychol Assess 2007; 19: 369-381, doi: 10.1037/1040-3590.19.4.369.

16. Beck AT, Ward $\mathrm{CH}$, Mendelson M, Mock J, Erbaugh J. An inventory for measuring depression. Arch Gen Psychiatry 1961; 4: 561-571, doi: 10.1001/archpsyc.1961.01710120031004.

17. Lykke J, Hesse M, Austin SF, Oestrich I. Validity of the BPRS, the BDI and the BAI in dual diagnosis patients. Addict Behav 2008; 33: 292-300, doi: 10.1016/j.addbeh. 2007.09.020.

18. Sato T. The Eysenck Personality Questionnaire Brief Version: factor structure and reliability. J Psychol 2005; 139: 545-552.

19. Liow RY, Walker K, Wajid MA, Bedi G, Lennox CM. The reliability of the American Knee Society Score. Acta Orthop Scand 2000; 71: 603-608, doi: 10.1080/000164700317362244.

20. Martimbianco AL, Calabrese FR, Iha LA, Petrilli M, Lira NO, Carneiro FM. Reliability of the "American Knee Society Score" (AKSS). Acta Ortop Bras 2012; 20: 34-38, doi: 10.1590/ S1413-78522012000100007.
21. Bohannon RW, DePasquale L. Physical Functioning Scale of the Short-Form (SF) 36: internal consistency and validity with older adults. J Geriatr Phys Ther 2010; 33: 16-18.

22. Gong L, Dong JY. Patient's personality predicts recovery after total knee arthroplasty: a retrospective study. $J$ Orthop Sci 2014; 19: 263-269, doi: 10.1007/s00776-013-0505-z.

23. Adie S, Dao A, Harris IA, Naylor JM, Mittal R. Satisfaction with joint replacement in public versus private hospitals: a cohort study. ANZ J Surg 2012; 82: 616-624.

24. Hanusch BC, O'Connor DB, lons P, Scott A, Gregg PJ. Effects of psychological distress and perceptions of illness on recovery from total knee replacement. Bone Joint $J$ 2014; 96-B: 210-216.

25. Wylde $\mathrm{V}$, Dixon $\mathrm{S}$, Blom AW. The role of preoperative selfefficacy in predicting outcome after total knee replacement. Musculoskeletal Care 2012; 10: 110-118, doi: 10.1002/msc. 1008.

26. Fisher DA, Dierckman B, Watts MR, Davis K. Looks good but feels bad: factors that contribute to poor results after total knee arthroplasty. J Arthroplasty 2007; 22: 39-42, doi: 10.1016/j.arth.2007.04.011.

27. Lim YC, Yobas P, Chen HC. Efficacy of relaxation intervention on pain, self-efficacy, and stress-related variables in patients following total knee replacement surgery. Pain Manag Nurs 2014; 15: 888-896, doi: 10.1016/j.pmn.2014.02.001.

28. Baumann C, Rat AC, Mainard D, Cuny C, Guillemin F. Importance of patient satisfaction with care in predicting osteoarthritis-specific health-related quality of life one year after total joint arthroplasty. Qual Life Res 2011; 20: 15811588, doi: 10.1007/s11136-011-9913-8.

29. Duivenvoorden T, Vissers MM, Verhaar JA, Busschbach JJ, Gosens T, Bloem RM, et al. Anxiety and depressive symptoms before and after total hip and knee arthroplasty: a prospective multicentre study. Osteoarthritis Cartilage 2013; 21: 1834-1840, doi: 10.1016/j.joca.2013.08.022.

30. Sharpley AL, Hockney R, McPeake L, Geddes JR, Cowen PJ. Folic acid supplementation for prevention of mood disorders in young people at familial risk: a randomised, double blind, placebo controlled trial. J Affect Disord 2014; 167: 306-311, doi: 10.1016/j.jad.2014.06.011.

31. Singh JA, Lewallen DG. Depression in primary TKA and higher medical comorbidities in revision TKA are associated with suboptimal subjective improvement in knee function. BMC Musculoskelet Disord 2014; 15: 127, doi: 10.1186/1471-2474-15-127.

32. Papakostidou I, Dailiana ZH, Papapolychroniou T, Liaropoulos L, Zintzaras E, Karachalios TS, et al. Factors affecting the quality of life after total knee arthroplasties: a prospective study. BMC Musculoskelet Disord 2012; 13: 116, doi: 10.1186/1471-247413-116.

33. Vissers MM, Bussmann JB, Verhaar JA, Busschbach JJ, Bierma-Zeinstra SM, Reijman M. Psychological factors affecting the outcome of total hip and knee arthroplasty: a systematic review. Semin Arthritis Rheum 2012; 41: 576-588, doi: 10.1016/j.semarthrit.2011.07.003.

34. Nunez M, Lozano L, Nunez E, Segur JM, Sastre S, Macule F, et al. Total knee replacement and health-related quality of life: factors influencing long-term outcomes. Arthritis Rheum 2009; 61: 1062-1069, doi: 10.1002/art.24644. 